\title{
The morphometry of the cavernous part of the internal carotid artery
}

\author{
M. Farımaz , H.H. Çelik' , K.M. Ergun' ${ }^{1}$, A. Akgöz², B. Urfalı ${ }^{3}$ \\ ${ }^{1}$ Department of Anatomy, Faculty of Medicine, Hacettepe University, Ankara, Turkey \\ ${ }^{2}$ Department of Radiology, Faculty of Medicine, Hacettepe University, Ankara, Turkey \\ ${ }^{3}$ Department of Neurosurgery, Faculty of Medicine, Mustafa Kemal University, Hatay, Turkey \\ [Received: 8 March 2018; Accepted: 23 April 2018]
}

Background: In the study, the morphometric evaluation of internal carotid artery (ICA) was performed in order to show the differences between the age groups and genders.

Materials and methods: In the study, descriptive measurements of intercarotid distance on the computed tomography of 173 (88 male [M], 85 female [F]) patients and the intercavernous distance on magnetic resonance images (MR/s) of 49 (19 M, 30 F) individuals were reviewed.

Results: Intercarotid distance was found to be close to the border of statistical significance and for results of the comparative measurements that were performed in the study; no significant sex-associated difference was observed for the distance between the gender and midpoint of the sella turcica and medial margin of the right ICA. Compared to gender, the distance between the base of the sella turcica and the base of the left ICA is found to be closed to of statistical significance. A statistically significant difference was obtained for the distance between the midpoint of sella turcica and medial margin of the left ICA and for the distance between the base of the sella turcica and the base of right ICA. Although it is observed that there is a weak correlation between the age and the distance between midpoint of the sella turcica and medial margin of the right ICA, statistically there is a significant difference between them.

Conclusions: Obtained results, planning of surgical interventions are supportive and guiding in terms of prevention of damage of to ICA in three dimensional thinking and operations. (Folia Morphol 2019; 78, 1: 54-62)

Key words: internal carotid artery, cavernous sinus, morphometry, transsphenoidal surgery

\section{INTRODUCTION}

The internal carotid artery (ICA) is one of the main arteries of the central nervous system. The cavernous part of this artery is important clinically in transsphenoidal surgical procedures. Transsphenoidal surgical procedures minimise the risk of complications and prevent bleeding of the vessel [12]. Transsphenoidal surgery and its modifications are important while taking biopsies from sellar and parasellar lesions
$[3,8,10,15,21,24]$. Bleeding of the ICA in the sellar region may be important for mortality and morbidities. Therefore, prior to surgical procedures it is clinically important to know the transverse distance between the right and left internal carotid arteries, which are found on both sides of the sella turcica [12].

The aim of this study is the morphometric analysis of the cavernous part of the ICA in an anatomically normal population. Additionally, the analysis was per- 
formed according to age groups and sex. We believe that this analysis will have a significant impact on planning surgical interventions in the region.

\section{MATERIALS AND METHODS}

\section{Patient population}

The morphometric analysis was performed in two groups. The first group consisted of patients who underwent brain computed tomography (CT) angiography examination in May, June and July 2015 in the Radiology Department of Hacettepe Medical Faculty. The second group consisted of patients who underwent three-dimensional (3D) contrast magnetic resonance (MR) examinations in 2015 in same department. The 201 patients who underwent CT angiography were between 40 and 71 years old. However, 28 of them were not included in the study because of presence of stents, stenosis, occlusion, plaques or technical reasons. The patients who underwent 3D contrast MR examination were between 21 and 84 years of age. This group included 50 patients, but one of the patients was excluded due to the presence of a meningioma. Ethical approval for the study was obtained from the ethics committee of our institution (December 16, 2015, GO 15/777-18).

\section{CT examination protocol}

Computed tomography angiographies of the patients were performed using dual $\mathrm{CT}$, which has a double tube system and 64 sections (Somatom Definition; Siemens Erlangen, Germany). The MR images (MRI) were taken in a 1.5 Tesla (Signa HDxT, GE Healthcare, USA) instrument. The acquisition details are as follows: section thickness was $1 \mathrm{~mm}, \mathrm{NEX} 1$, matrix $256 \times 256$, TR-10 ms, TE-4.2 ms, T1-450, FA-15, sequence 3D T1 FSPGR.

\section{Image evaluation and analysis}

The measurements were taken in sagittal, coronal and axial sections of $\mathrm{CT}$ angiographies and $3 \mathrm{D}$ contrast MRI. In the sagittal axis, the deepest part of the sella turcica was chosen as a reference point for the measurements. The measured sections of the MRI were taken post contrast in axial and sagittal planes and were in 3D. In these images, the morphometric analysis was done in a plane vertical to the sagittal base and coronal reformats were obtained. The distances measured in the $\mathrm{CT}$ angiographies were as follows: in the first sagittal axis obtained, the deepest section of the sella turcica was found. This axis was used in every measured parameter. In the axial sections, the intercarotid distance (the nearest point of the medial borders of the internal carotid arteries) was measured. In the coronal sections, the mid-point of the sella turcica (determined by measuring the distance between the carotid sulci on each side and dividing this value by two) and the distance between the medial borders of the internal carotid arteries were measured. In the coronal sections, the distance between the base of the sella turcica and the bases of the internal carotid arteries in the cavernous sinus was calculated.

In the 3D contrast MRI, the distance between the medial borders of the cavernous sinus was measured. The measurements were taken by obtaining the coronal reformats in a plane that is vertical to the base of the sella turcica.

\section{Statistical analysis}

The obtained data were analysed statistically using the SPSS 22 software. The correlations between the different parameters were determined with the Pearson test. For the comparison of the parameters between two groups Student's t test was used. Beforehand the usage of Student's t test, a normality was assessed for the statistical analysis. In all the statistical analyses, a p value under 0.05 was considered statistically significant.

\section{RESULTS}

In the study, the intercarotid distance was measured on CT scans of 173 patients (88 males [M], 85 females [F]) and the intercavernous distance was measured in the MRI of 49 patients (19 M, $30 \mathrm{~F}$ ). Additionally, comparative measurements were done on CT brain scans. These comparative measurements included the distance between the medial borders of the right and left internal carotid arteries and the midpoint of the sella turcica and the distance between the base of sella turcica and the bases of the right and left internal carotid arteries (Table 1).

The obtained data showed that there was a strong correlation between the intercarotid distance and the distance between the mid-point of the sella turcica and the medial borders of the right and left internal carotid arteries $(p<0.001)$. There was a weak correlation between the intercarotid distance and the distance between the base of sella turcica and base of the right ICA. However, in this group, statistically significant differences were present between 
Table 1. Statistical evaluation results according to age and gender groups of measured distances

\begin{tabular}{lccccc}
\hline & D1 & D2 & D3 & D4 & D5 \\
\hline Gender $(\mathrm{n}=173)$ & & & & \\
$\quad$ Female $(\mathrm{n}=85)$ & $15.8 \pm 38$ & $9 \pm 2.3$ & $8.8 \pm 1.8$ & $0.2 \pm 1.7$ & $0 \pm 2$ \\
$\quad$ Male $(\mathrm{n}=88)$ & $17.1 \pm 4.7$ & $9.6 \pm 2.5$ & $9.7 \pm 2.7$ & $-0.6 \pm 2.10$ & $0 \pm 2$ \\
P & $0.051^{*}$ & 0.111 & $0.014^{*}$ & $0.006^{*}$ & 0.07 \\
Age groups & & & & \\
$\quad \leq 40(\mathrm{n}=26)$ & $15 \pm 3.9$ & $8.3 \pm 2$ & $8.7 \pm 1.9$ & $-0.5 \pm 1.8$ & $0 \pm 2$ \\
$41-50(\mathrm{n}=29)$ & $16.3 \pm 4.7$ & $9 \pm 2.7$ & $9.1 \pm 3.1$ & $-0.7 \pm 1.8$ & $0 \pm 2$ \\
$51-60(\mathrm{n}=40)$ & $17.2 \pm 4.1$ & $9.4 \pm 2.2$ & $9.7 \pm 2.2$ & $-0.5 \pm 17$ & $0 \pm 2$ \\
$61-70(\mathrm{n}=35)$ & $17.1 \pm 4.7$ & $9.6 \pm 2.5$ & $9.63 \pm 2.2$ & $0.4 \pm 2.2$ & $0 \pm 2$ \\
$\geq 71(\mathrm{n}=43)$ & $16.4 \pm 4.2$ & $9.7 \pm 2.3$ & $9.0 \pm 2.2$ & $0 \pm 2.1$ & $0 \pm 2$ \\
\hline
\end{tabular}

Data is shown as mean \pm standard deviation. D1 — intercarotid distance $(\mathrm{mm}), \mathrm{D} 2$ - in coronal sections, the distance between the mid-point of sella turcica and medial border of right internal carotid arteries coursing in cavernous sinus $(\mathrm{mm}), \mathrm{D} 3$ - in coronal sections, the distance between the mid-point of the sella turcica and the medial border of the left internal carotid artery coursing in the cavernous sinus $(\mathrm{mm}), \mathrm{D} 4$ - in coronal sections, the distance between the base of the sella turcica and the base of the right internal carotid artery coursing in cavernous sinus (mm), D5 - in coronal sections, the distance between the base of the sella turcica and the base of the left internal carotid artery coursing in cavernous sinus (mm); $p>0.05$ - statistically not significant difference; ${ }^{*} p<0.05$ - statistically significant difference

Table 2. Correlation analysis test results

\begin{tabular}{lcc}
\hline Distances & $\mathbf{r}$ & $\mathbf{p}$ \\
\hline D1-D2 & 0.797 & $<0.001^{*}$ \\
D1-D3 & 0.829 & $<0.001^{*}$ \\
D1-D4 & -0.272 & $<0.001^{*}$ \\
D1-D5 & -0.413 & $<0.001^{*}$ \\
D2-D3 & 0.614 & $<0.001^{*}$ \\
D2-D4 & -0.154 & $0.043^{*}$ \\
D2-D5 & -0.192 & $0.012^{*}$ \\
D3-D5 & -0.228 & $0.003^{*}$ \\
D4-D5 & 0.552 & $<0.001^{*}$ \\
\hline
\end{tabular}

$\mathrm{r}$ - correlation coefficient; other abbreviations - see Table 1; * $\mathrm{p}<0.05$ : statistically significant

the distances $(p<0.001)$. Although, there was only a mild degree of correlation between the intercarotid distance and the distance between the base of the sella turcica and the base of the left ICA ( $p<0.001)$, the measured distances showed statistically significant differences in this group. Additionally, an inverse correlation was detected between the intercarotid distance and the distance between the base of the sella turcica and the bases of the right and the left internal carotid arteries. There was a mild degree of correlation between the mid-point of the sella turcica and the medial border of the right ICA and between the mid-point of the sella turcica and the medial border of the left ICA ( $p<0.001)$. In this group, the measured distances showed statistically significant differences. There were weak correlations between the mid-point of the sella turcica and the medial bor- der of the right ICA and between the base of the sella turcica and bases of the right and the left internal carotid arteries. However, in these groups, the measured distances showed statistically significant differences. There were mild degree of correlations between the distance of the base of the sella turcica and the base of the right ICA and the distance between the base of the sella turcica and the left ICA $(p<0.001)$. There were statistically significant differences among the distances in these groups (Table 2).

Additionally, the types of sphenoidal sinuses were also examined in the study. Of the 173 cases, $86.7 \%$ had sellar type, $10.98 \%$ had presellar type, $2.31 \%$ had conchal type sinuses. There was no statistically significant difference between the types of sphenoidal sinuses and measured distances ( $p>0.05)$.

The mean value of the intercarotid distance was $13.6 \pm 2.8 \mathrm{~mm}$ in both sexes (Fig. 1). In females, the mean value was $14.1 \pm 2.8 \mathrm{~mm}$ and in males, it was $13.0 \pm 2.8 \mathrm{~mm}$ (Table 3 ). The mean value of the intercarotid distance was $16.5 \pm 4.3 \mathrm{~mm}$ in both sexes (Figs. 2, 3). In females, the mean value was $15.8 \pm 3.8$ $\mathrm{mm}$, and in males, it was $17.1 \pm 4.7 \mathrm{~mm}$ (Table 1). The mean value of the intercavernous distance was $13.6 \pm 2.8 \mathrm{~mm}$ of both sexes (Fig. 1). In the statistical analysis for differences between females and males, the intercarotid distance was found to be close to the significance level $(p=0.051)$ and the intercavernous distance was found to be non-significant ( $p>0.05$ ). Additionally, the intercarotid distance did not show any correlation with age and the intercavernous distance showed a negative correlation with age $(r=-0.079)$. 


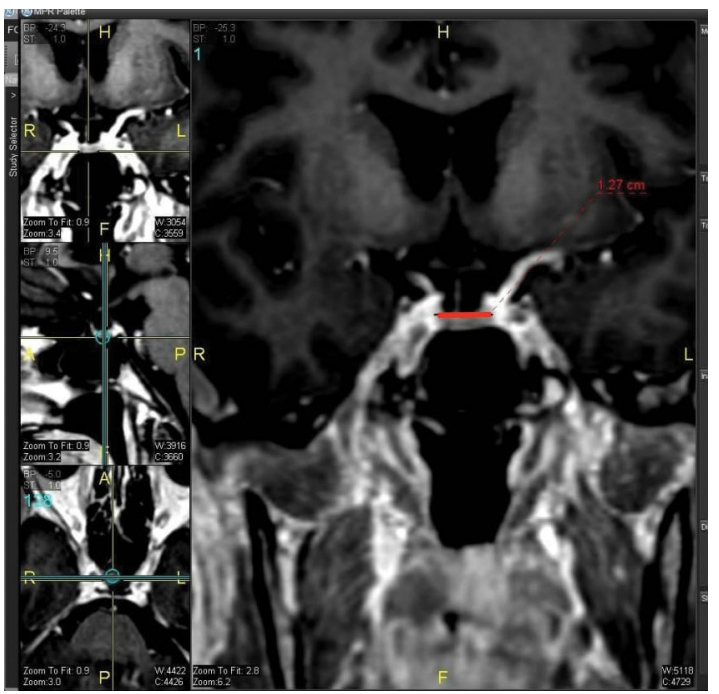

Figure 1. The measurement of intercavernous distance (magnetic resonance).

Table 3. Intercavernous distance

\begin{tabular}{lccc}
\hline Intercavernous distance & Mean \pm SD & Min-Max & $\mathbf{p}$ \\
\hline Female $(\mathrm{n}=30)$ & $14.1 \pm 2.8$ & $7.8 / 21.5$ & \\
Male $(\mathrm{n}=19)$ & $13.0 \pm 2.8$ & $7.8 / 18.6$ & \\
Sex $(\mathrm{n}=49)$ & $13.6 \pm 2.8$ & $7.8 / 21.5$ & 0.183 \\
Age & $56.5 \pm 12.8$ & $24 / 84$ & \\
\hline
\end{tabular}

p > 0.05 - statistically not significant difference; Min — minimum; Max — maximum; $\mathrm{SD}$ - standard deviation

The mean value of the distance between the midpoint of the sella turcica and the right medial border of the ICA coursing in the cavernous sinus was $9.3 \pm 2.4 \mathrm{~mm}$. In females, it was $9.0 \pm 2.3 \mathrm{~mm}$, and in males, it was $9.6 \pm 2.5 \mathrm{~mm}$. The mean value of the distance in between the mid-point of the sella turcica and the left internal carotid artery's medial border coursing in the cavernous sinus was $9.3 \pm 2.3 \mathrm{~mm}$. In females, it was $8.8 \pm 1.8 \mathrm{~mm}$ and, in males, it was $9.7 \pm 2.7 \mathrm{~mm}$ (Fig. 4)

The mean value of the distance between the base of the sella turcica and the base of the right ICA was $-0.2 \pm 2 \mathrm{~mm}$. In females, it was $0.2 \pm 1.7 \mathrm{~mm}$, and in males, it was $-0.6 \pm 2.1 \mathrm{~mm}$. The mean value of the distance between the base of the sella turcica and base of the left ICA was $0 \pm 2 \mathrm{~mm}$ (Fig. 5). In both sexes, the mean value was found to be $0 \pm 2 \mathrm{~mm}$. In the measurement of the distance between the base of the sella turcica and base of the right and left internal carotid arteries coursing in the cavernous sinus; the value was recognised as $(+)$ when the base of ICA was over the base of the sella turcica. When the base

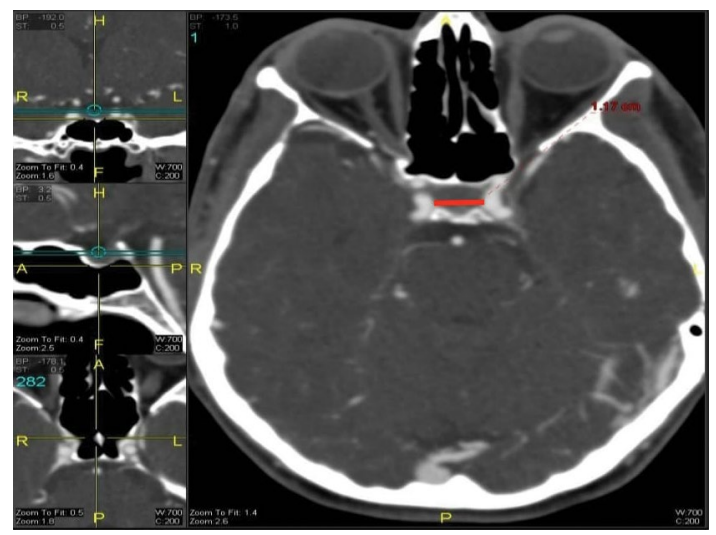

Figure 2. The measurement of intercarotid distance (computed tomography angiography).

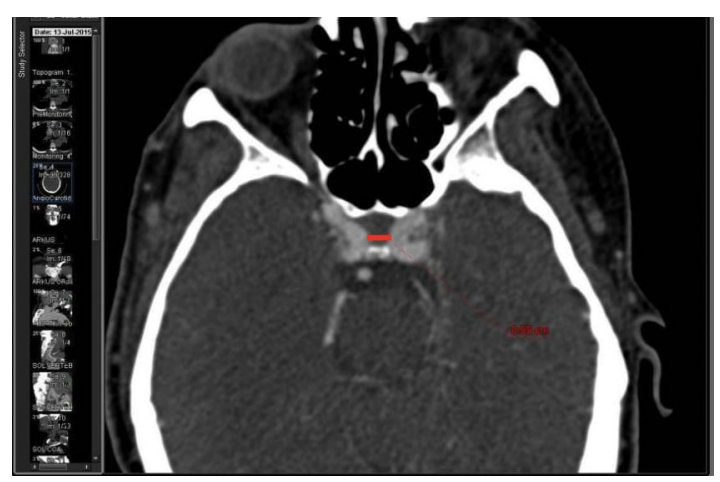

Figure 3. The minimum intercarotid distance measurement (computed tomography angiography).

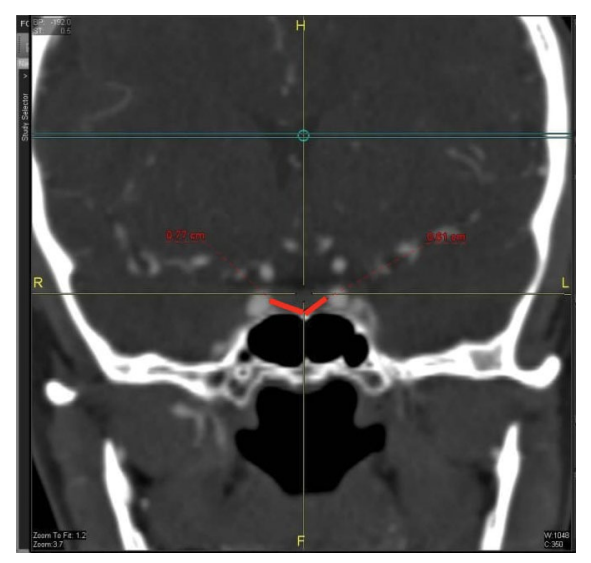

Figure 4. The measurement of the distance between the mid-point of the sella turcica and the medial borders of the right and left internal carotid artery (computed tomography angiography).

of the ICA was under the base of the sella turcica; it was recognised as (-) (Fig. 5).

In the statistical analysis of the comparative measurements between males and females, the distance 


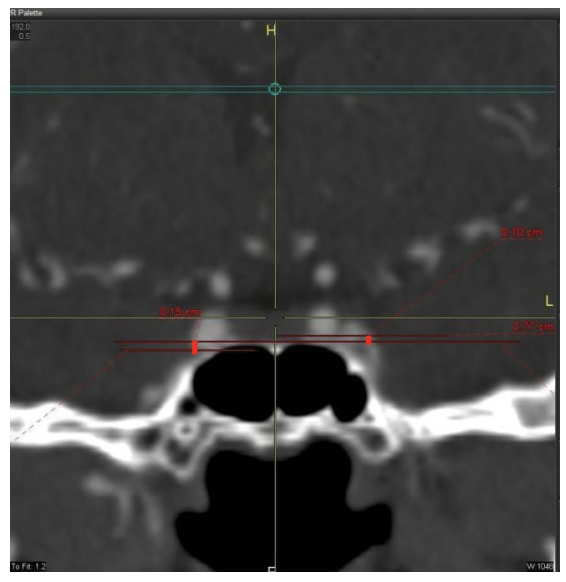

Figure 5. The measurement of the distance between the base of the sella turcica and the bases of the right and left internal carotid arteries (computed tomography angiography).

between the base of the sella turcica and the base of the left ICA was found to be close to the significance level ( $p=0.07$; Fig. 5 ). There was no statistically significant difference in the measurement of the distance between the mid-point of the sella turcica and the medial border of the right ICA. However, statistically significant differences were observed in the measurements of distances between mid-point of the sella turcica and the medial border of the ICA, between the mid-point of the sella turcica and the medial border of the left ICA and between the base of sella turcica and the base of right ICA ( $p=0.014$, $p=0.006$; Figs. 4, 5). Additionally, there was a weak correlation in the distance between mid-point of the sella turcica and the medial border of the right ICA with age $(r=0.186)$.

In the statistical analysis of the sex parameter between MR and CT groups, $\chi^{2}$ test was used. No statistically significant difference was observed between these two groups according to sex $(p=0.14)$. In the statistical analysis of the age parameter between MR and CT groups, Mann-Whitney $U$ test was used. No statistically significant difference was observed between these two groups according to age $(U=3926.5$, $p=0.43)$.

\section{DISCUSSION}

The intercarotid distance between the cavernous parts of the internal carotid arteries, sellar region, cavernous sinus, and their relation to each other are important clinically to prevent arterial bleeding during surgical procedures [1, 2, 5-7, 12, 19, 21, 22, 27]. The ICA is located on the medial side of the cavernous sinus
Table 4. Intercarotid distance (ID) at the level of the sella turcica

\begin{tabular}{lc}
\hline Authors & Mean ID [mm] \\
\hline Fujii et al. (1979) & 17 \\
Abuzayed et al. (2010) & 18 \\
Perondi et al. (2013) & 18 \\
Aktas et al. (2013) & 15.33 \\
Our study (2016) & 16.5 \\
\hline
\end{tabular}

(C4 segment) $[13,14]$. As there are no cranial nerves in the medial part of the cavernous sinus, ICA is the major structure encountered in transsphenoidal surgery.

In surgical procedures, after the exposure to the base of the sella turcica, the dura mater covering the hypophysis is defined. The dura mater splits into two layers. The periosteal dura forms the first layer and is related to the bone. After covering the base of the sella turcica, the periosteal dura courses to the lateral side and forms the medial wall of the cavernous sinus. Later, it continues as the base of the sinus. The dural layer, which is adherent to the hypophysis separates the gland from the medial side of the cavernous sinus. This layer continues as the lateral layer on the lateral side and roof of the cavernous sinus [25]. Therefore, the medial wall of the cavernous sinus is divided into two parts. In the upper section, the medial wall of the cavernous sinus is formed as the dural layer of the hypophysis without a bony support. In the lower section, the medial wall of the cavernous sinus is formed by the periosteal dura of the sella turcica and supported by the bony lateral wall of the sphenoidal sinus. Destrieux et al. [9] studied the microanatomy of the dura layers in the sellar region. Their definitions were in accordance with Romano et al. [21], the mean distance between the medial walls of the cavernous sinus was found to be $14.9 \mathrm{~mm}(10.1 / 18.22, \mathrm{n}=20)$ in their study.

In all the measurements done in our study, the sagittal axis, in which the deepest point of sella turcica was defined, was used first. The deepest point of sella turcica can best be observed and measured in CT images taken in the sagittal axis and, therefore, this axis was used for the morphometric examination. In studies performed on cadavers, Fujii et al. [11], (at the level of the sellar base), Abuzayed et al. [1] (at the sellar level), Perondi et al. [19] (at level of the sellar base), and Aktas et al. [2] (at level of the dorsum sellae) measured the intercarotid distances. It was observed that the measurements done on CT scans were in accordance with the cadaver measurements as shown in Table 4. 
Table 5. Comparison of the measured intercarotid distances (ID)

\begin{tabular}{llcc}
\hline & Researchers & ID [mm] & No. of cases \\
\hline Cadaver & Fuji (1979) & 17 & 25 \\
& Ozcan (2010) & 13.33 & 29 \\
& Abuzayed (2010) & $13-22$ & 30 \\
& Aktas (2013) & 15.33 & 28 \\
& Perondi (2013) & 18 & 30 \\
Magnetic resonance & Cebula (2014) & 12.15 & 20 \\
& Scotti (1988) & 16.60 & 25 \\
& Knappe (2009) & 17.8 & 117 \\
Computed tomography angiography & Zada (2011) & 16.2 & 100 \\
& Sasagawa (2013) & 19.4 & 138 \\
& Zhang (2012) & 20.6 & 96 \\
\hline
\end{tabular}

In our study, in the measurements done on the $\mathrm{MRI}$, the mean intercavernous distance was found to be $14.1 \pm 2.8 \mathrm{~mm}$ in females and $13.0 \pm 2.8 \mathrm{~mm}$ in males. There was no statistically significant difference between males and females for this measurement $(p=0.183$ ). Additionally, the intercavernous distance showed a negative correlation with age $(r=-0.079)$. The mean value of the intercavernous distance was found to be $14.9 \mathrm{~mm}$ by Romano et al. [21], $12.7 \mathrm{~mm}$ by Zada et al. [27]. However, in our study, we found this distance to be $13.6 \mathrm{~mm}$.

In this study, in the measurements done on the CT scans, the mean value of the nearest distance of medial walls of the internal carotid arteries coursing in the cavernous sinus was found to be $15.8 \pm$ $\pm 3.8 \mathrm{~mm}$ in females and $17.1 \pm 4.7 \mathrm{~mm}$ in males. In surgical approaches, the medial wall of the ICA will have to be penetrated before an injury could occur to the ICA coursing in the cavernous sinus. Therefore, if abundant venous bleeding is observed during a transsphenoidal surgery, it is likely to be secondary to the penetration of the cavernous sinus and surgeons must be aware of the course of the ICA, which is approximately $3 \mathrm{~mm}$ away. The study also showed a negative correlation between age and the mean distance between the medial walls of the internal carotid arteries. All these findings are important clinically.

In our study, the CT scan data showed that the measurement of the intercarotid distance of the cavernous part of the ICA was $16.5 \mathrm{~mm}$ (mean value). The intercarotid distance was measured in the ax- ial sections of $C T$ images. In MRI, studies found in literature measured this distance to be $16.6 \mathrm{~mm}$ in Scotti et al.'s study [23], $17.8 \mathrm{~mm}$ in Knappe et al.'s research [16], $16.2 \mathrm{~mm}$ in Zada et al.'s work [27], $20.6 \mathrm{~mm}$ in Zhang et al.'s manuscript [28] and $19.4 \mathrm{~mm}$ in Sasagawa et al.'s study [22]. The mean value of the intercarotid distance in cadavers was found to be $17 \mathrm{~mm}$ by Fujii et al. [11], $13.33 \mathrm{~mm}$ by Ozcan et al. [18], $18 \mathrm{~mm}$ by Abuzayed et al. [1], $18 \mathrm{~mm}$ by Perondi et al. [19], $15.33 \mathrm{~mm}$ by Aktas et al. [2], and $12.15 \mathrm{~mm}$ by Cebula et al. [5] (Table 5).

As shown in Table 5, measurement of the intercarotid distance was primarily done on cadavers. Similarity in the measured intercarotid distances between cadavers and living patients suggests that radiologic methods can also be used accurately to obtain these measurements. Comparatively, our study had the highest number of patients in whom the measurements were done.

Gupta [12] and Yilmazlar et al. [26] measured the distances from three different views: anterior, medial and posterior. According to Gupta [12], the nearest distance between the internal carotid arteries was found at the maximum sellar depth. Yilmazlar et al. [26] reported a statistically significant difference in the medial and posterior intercarotid distances in studies done on MRI and cadavers. Zhang et al. [28] and Carrabba et al. [4] measured the intercarotid distances on CT scan and found them as to be $20.6 \mathrm{~mm}$ and $16.65 \mathrm{~mm}$, respectively. The main difference between our study and these two studies was the large size of our patient population. 
If the intercarotid distance between the cavernous parts of the internal carotid arteries is less than $10 \mathrm{~mm}$, transsphenoidal surgery will be difficult [20]. In our study, the minimum intercarotid distance was found to be $5.5 \mathrm{~mm}$. Although, the short intercarotid distance does not make the operation impossible, it can only be performed by competent surgeons. In literature, the mean intercarotid distance of internal carotid arteries coursing in the cavernous sinus was found to be $12 \mathrm{~mm}$ (4-18 $\mathrm{mm}$ ). However, the artery may be tortuous in the carotid sulcus or more laterally located in the sinus [17]. In our series, the maximum intercarotid distance was found to be $27.3 \mathrm{~mm}$. However, this value does not create a contraindication for transsphenoidal surgery [26].

The data in Table 5 show the various studies done on this subject. However, in our study, we also measured the distances between the internal carotid arteries and the sella turcica, in addition to the intercarotid and intercavernous distances.

In our study, the distances between the mid-point of the sella turcica and the right and left internal carotid arteries were measured. These measurements were performed in the coronal sections of $\mathrm{CT}$ images. On the right side, it was $9.0 \pm 2.3 \mathrm{~mm}$ in females and $9.6 \pm$ $\pm 2.5 \mathrm{~mm}$ in males. This distance did not show any statistically significant sex-related difference $(p=0.111)$.

The mean distance between mid-point of the sella turcica and the medial border of the left ICA coursing in the cavernous sinus was $8.8 \pm 1.8 \mathrm{~mm}$ in females and $9.7 \pm 2.7 \mathrm{~mm}$ in males. There was a statistically significant sex-related difference for this distance $(p=0.014)$. The mean distance in between the mid-point of the sella turcica and the medial border of the right ICA coursing in the cavernous sinus showed a weak correlation with age groups. These findings will be important during surgical procedures.

Cheng et al. [7] studied 144 CT scans (75 females and 69 males). In the coronal plane, the mean distance of the ICA to the mid-point of the sella turcica was found to be $11.25 \mathrm{~mm}$ on the right and $11.06 \mathrm{~mm}$ on the left side. Cheng et al. [7] measured the distance between the mid-point of the posterior vertical segment of the ICA and the transverse line drawn from the mid-point of the sella turcica. It was $6.41 \mathrm{~mm}$ on the right and $6.31 \mathrm{~mm}$ on the left side. They did not observe any statistically significant difference between right and left $(p>0.05)$. In our study, the distance between the mid-point of the sella turcica and the medial borders of the right and left internal carotid arteries coursing in the cavernous sinus was measured directly from the mid-point of the sella turcica using an oblique coursing measurement method. In our study, there was no statistically significant difference in the measurement between the mid-point of the sella turcica and the medial border of the right ICA between males and females $(p=0.111)$. However, the measurement between the mid-point of the sella turcica and the medial border of the left ICA showed a statistically significant difference between males and females $(p=0.014)$.

Cheng et al. [6] studied the relationships of the optic canal and the ICA on CT scans of 200 patients in the coronal, sagittal, and axial planes. In two of their measurements, they used the sagittal axis, in which the sella turcica is deepest, which is similar to our study. In these two measurements, they drew a vertical plane to the sellar base in the axial plane and measured the distance between the right and left internal carotid arteries. The mean value was $8.87 \mathrm{~mm}$ on the right and $8.94 \mathrm{~mm}$ on the left side. In another measurement, they drew a vertical line to the sellar base where the cavernous part of the posterior vertical segment of the ICA made the largest bulge. They measured the distance between this line and the right and left internal carotid arteries. The mean distance was $11.34 \mathrm{~mm}$ on the right and $11.08 \mathrm{~mm}$ on the left side. They did not find any statistically significant difference between the right and left sides $(p>0.05)$. In our study, there was a statistically significant difference in the measurement of the distance between the mid-point of the sella turcica and the medial border of the left ICA coursing in the cavernous sinus between males and females. The observations from our study were different from those made by Cheng et al. [6]. In our study, the measured parameters were different and the obtained data were analysed according to age and sex.

Another parameter measured in our study was the distance between the base of the sella turcica and the bases of the right and left internal carotid arteries coursing in the cavernous sinus. These measurements were performed in the coronal sections of $\mathrm{CT}$ images. The mean value of the distance between the base of the sella turcica and the base of the right ICA was $0.2 \pm 1.7 \mathrm{~mm}$ in females and $-0.6 \pm 2.1 \mathrm{~mm}$ in males. There was a statistically significant difference between males and females in this measurement 
$(p=0.006)$. This distance did not show any correlation with age in our study. The mean value of the distance between the base of the sella turcica and the base of the left ICA was $0 \pm 2 \mathrm{~mm}$ in males and females. This distance was close to being statistically significant both in males and females $(p=0.07)$. These measurements will be important during surgical procedures while the surgeon is working with the microscope or endoscope. During transsphenoidal surgery, the distance between the base of the sella turcica and the base of the ICA is very important while penetrating to the intrasellar region. There were no data on this finding in literature.

Surgeons must remember the importance of very small differences in the intercarotid and intercavernous distances. The measurement of the distance between the mid-point of the sella turcica and the medial borders of the right and left internal carotid arteries coursing in the cavernous sinus will be very important during angulations to either the left or right sides for a surgeon to be most successful. The distance between the base of the sella turcica and the bases of the right and left internal carotid arteries coursing in the cavernous sinus will be important for the angulation by the surgeon after defining the base of the sella turcica.

\section{CONCLUSIONS}

In this study, some of the important anatomic measurements were determined in order to allow surgeons to use new approaches during transsphenoidal surgical procedures. In vivo radiologic measurements were also performed. The data obtained by the comparison of these measurements will be very important during the planning of surgical procedures and while thinking three dimensionally. With the help of these measurements, the ICA will be better protected during surgical manipulations.

\section{Acknowledgements}

The authors would like to thanks Prof. Mustafa F. Sargon, who is the head of the Anatomy Department, Hacettepe University Faculty of Medicine for his invaluable assistance and encouragement in performing of this study. Thanks Prof. Mutlu Hayran, at Department of Preventive Oncology, Hacettepe University Faculty of Medicine, for his assistance with the statistical analysis and thanks Department of Radiology, Hacettepe University Faculty of Medicine for radiological images which are used in this study.

\section{REFERENCES}

1. Abuzayed B, Tanriover N, Gazioglu N, et al. Endoscopic anatomy and approaches of the cavernous sinus: cadaver study. Surg Radiol Anat. 2010; 32(5): 499-508, doi: 10.1007/ s00276-010-0651-3, indexed in Pubmed: 20443000.

2. Aktas U, Yilmazlar S, Ugras N. Anatomical restrictions in the transsphenoidal, transclival approach to the upper clival region: a cadaveric, anatomic study. J Craniomaxillofac Surg. 2013; 41(6): 457-467, doi: 10.1016/j. jcms.2012.11.011, indexed in Pubmed: 23257317.

3. Arai H, Sato K, Okuda O, et al. Transcranial transsphenoidal approach for tuberculum sellae meningiomas. Acta Neurochir (Wien). 2000; 142(7): 751-6; discussion 756, indexed in Pubmed: 10955669.

4. Carrabba G, Locatelli M, Mattei L, et al. Transphenoidal surgery in acromegalic patients: anatomical considerations and potential pitfalls. Acta Neurochir (Wien). 2013; 155(1): 125-130, doi: 10.1007/s00701-012-1527-6, indexed in Pubmed: 23180167.

5. Cebula H, Kurbanov A, Zimmer LA, et al. Endoscopic, endonasal variability in the anatomy of the internal carotid artery. World Neurosurg. 2014; 82(6): e759e764, doi: 10.1016/j.wneu.2014.09.021, indexed in Pubmed: 25238676.

6. Cheng $Y$, Liu M, Zhang S, et al. Optic canal (OC) and internal carotid artery (ICA) in sellar region. Surg Radiol Anat. 2013; 35(9): 797-801, doi: 10.1007/s00276-013-1193-2, indexed in Pubmed: 24005376.

7. Cheng Ye, Zhang H, Su L, et al. Anatomical study of cavernous segment of the internal carotid artery and its relationship to the structures in sella region. J Craniofac Surg. 2013; 24(2): 622-625, doi: 10.1097/ SCS.0b013e3182801f30, indexed in Pubmed: 23524760.

8. Couldwell WT, Weiss MH, Rabb C, et al. Variations on the standard transsphenoidal approach to the sellar region, with emphasis on the extended approaches and parasellar approaches: surgical experience in 105 cases. Neurosurgery. 2004; 55(3): 539-547, indexed in Pubmed: 15335421.

9. Destrieux C, Kakou MK, Velut S, et al. Microanatomy of the hypophyseal fossa boundaries. J Neurosurg. 1998; 88(4): 743-752, doi: 10.3171/jns.1998.88.4.0743, indexed in Pubmed: 9525722.

10. Fahlbusch R, Thapar K. New developments in pituitary surgical techniques. Baillieres Best Pract Res Clin Endocrinol Metab. 1999; 13(3): 471-484, indexed in Pubmed: 10909437.

11. Fujii K, Chambers SM, Rhoton AL. Neurovascular relationships of the sphenoid sinus. A microsurgical study. J Neurosurg. 1979; 50(1): 31-39, doi: 10.3171/ jns.1979.50.1.0031, indexed in Pubmed: 758376.

12. Gupta T. An anatomical study of inter carotid distances in the sellar region with a surgical perspective. J Morphol Sci. 2009; 26: 23-26.

13. Hakuba A, Tanaka K, Suzuki T, et al. A combined orbitozygomatic infratemporal epidural and subdural approach for lesions involving the entire cavernous sinus. J Neurosurg. 1989; 71(5 Pt 1): 699-704, doi: 10.3171/ jns.1989.71.5.0699, indexed in Pubmed: 2809723.

14. Harris FS, Rhoton AL. Anatomy of the cavernous sinus. A microsurgical study. J Neurosurg. 1976; 45(2): 
169-180, doi: 10.3171/jns.1976.45.2.0169, indexed in Pubmed: 939976.

15. Kitano M, Taneda M. Extended transsphenoidal approach with submucosal posterior ethmoidectomy for parasellar tumors. Technical note. J Neurosurg. 2001; 94(6): 999-1004, doi: 10.3171/jns.2001.94.6.0999, indexed in Pubmed: 11409533.

16. Knappe UJ, Jaursch-Hancke C, Schönmayr R, et al. Assessment of normal perisellar anatomy in $1.5 \mathrm{~T}$ T2-weighted MRI and comparison with the anatomic criteria defining cavernous sinus invasion of pituitary adenomas. Cent Eur Neurosurg. 2009; 70(3): 130-136, doi: 10.1055/s-0029-1216363, indexed in Pubmed: 19701871.

17. Lang J. Clinical Anatomy of the Head: Neurocranium, Orbit, Craniocervical Regions, 6th ed. Springer Verlag, Berlin 1983: 200-201.

18. Ozcan T, Yilmazlar S, Aker S, et al. Surgical limits in transnasal approach to opticocarotid region and planum sphenoidale: an anatomic cadaveric study. World Neurosurg. 2010; 73(4): 326-333, doi: 10.1016/j.wneu.2010.01.015, indexed in Pubmed: 20849787.

19. Perondi GE, Isolan GR, de Aguiar PH, et al. Endoscopic anatomy of sellar region. Pituitary. 2013; 16(2): 251-259, doi: 10.1007/s11102-012-0413-9, indexed in Pubmed: 22847021.

20. Renn WH, Rhoton AL. Microsurgical anatomy of the sellar region. J Neurosurg. 1975; 43(3): 288-298, doi: 10.3171/ jns.1975.43.3.0288, indexed in Pubmed: 1151464.

21. Romano A, Zuccarello M, van Loveren HR, et al. Expanding the boundaries of the transsphenoidal approach: a microanatomic study. Clin Anat. 2001; 14(1): 1-9, doi: 10.1002/1098-2353(200101)14:1<1::AID-CA1000>3.0. CO;2-3, indexed in Pubmed: 11135390.
22. Sasagawa $Y$, Tachibana O, Doai M, et al. Internal carotid arterial shift after transsphenoidal surgery in pituitary adenomas with cavernous sinus invasion. Pituitary. 2013; 16(4): 465-470, doi: 10.1007/s11102-013-0492-2, indexed in Pubmed: 23720159.

23. Scotti G, Yu CY, Dillon WP, et al. MR imaging of cavernous sinus involvement by pituitary adenomas. AJR Am J Roentgenol. 1988; 151(4): 799-806, doi: 10.2214/ajr.151.4.799, indexed in Pubmed: 3262283.

24. Spencer WR, Levine JM, Couldwell WT, et al. Approaches to the sellar and parasellar region: a retrospective comparison of the endonasal-transsphenoidal and sublabial-transsphenoidal approaches. Otolaryngol Head Neck Surg. 2000; 122(3): 367-369, doi: 10.1016/S01945998(00)70050-7, indexed in Pubmed: 10699812.

25. Umansky F, Nathan $\mathrm{H}$. The lateral wall of the cavernous sinus. With special reference to the nerves related to it. J Neurosurg. 1982; 56(2): 228-234, doi: 10.3171/ jns.1982.56.2.0228, indexed in Pubmed: 7054432.

26. Yilmazlar S, Kocaeli $\mathrm{H}$, Eyigor $\mathrm{O}$, et al. Clinical importance of the basal cavernous sinuses and cavernous carotid arteries relative to the pituitary gland and macroadenomas: quantitative analysis of the complete anatomy. Surg Neurol. 2008; 70(2): 165-74; discussion 174, doi: 10.1016/j. surneu.2007.06.094, indexed in Pubmed: 18262607.

27. Zada G, Agarwalla PK, Mukundan S, et al. The neurosurgical anatomy of the sphenoid sinus and sellar floor in endoscopic transsphenoidal surgery. J Neurosurg. 2011; 114(5): 1319-1330, doi: 10.3171/2010.11.JNS10768, indexed in Pubmed: 21235317.

28. Zhang $Y$, Tian $Y$, Song J, et al. Internal carotid artery in endoscopic endonasal transsphenoidal surgery. J Craniofac Surg. 2012; 23(6): 1866-1869, doi: 10.1097/ SCS.0b013e31826bf22a, indexed in Pubmed: 23172428. 